\title{
Expression of factors regulating cytokinesis in Bowen's disease
}

\author{
Hirofumi Okada, Mayumi Komine, Hidetoshi Tsuda, Koji Kamiya, Meijuan Jin, \\ Mamitaro Ohtsuki \\ Jichi Medical University, Japan
}

\section{Background}

Bowen's disease is a common type of squamous carcinoma in situ, which affects full thickness of the epidermis, but sometimes shows pagetoid growth. One of the characteristic histopathological features is the formation of multinucleated cells, called clumping cells, however, the mechanism of multinucleation has not been elucidated.

Multinucleation of the cell means a defect in cytokinesis, i.e., the division of the cytoplasm of the cell. Several molecules are involved in cytokinesis, such as Ras homologue gene family, member A (RhoA), epithelial cell transforming 2 (Ect2), and Rho-associated, coiled-coil containing protein kinase 1 (ROCK1). Knockdown of RhoA or Ect2 induces increase in multinucleated cells in culture $(1,2)$. Very recently, we have found that knockdown of IL-33 caused increased number of binucleated cells in cultured normal human epidermal keratinocytes (NHEKs) (paper in preparation).

IL-33 is one of Th2 type cytokines, produced by endothelial cells and epithelial cells, reported to be involved in the pathogenesis of atopic dermatitis and bronchial asthma(3). IL-33 resides also in the nucleus and works as a nuclear factor, but the precise role of nuclear IL-33 has not been fully elucidated(4). We have recently found that knockdown of IL33 in NHEKs caused increased number of binucleated cells, which also caused decrease in RhoA activation and the Ect2 expression.

Thus we tried to investigate the expression of IL-33, RhoA and Ect2 in Bowen's disease to explore the mechanism of multinucleation in Bowen's disease.

\section{Materials and Methods}

Formalin-fixed, paraffin-embedded samples from the lesion of Bowen's disease (32cases) and Bowenoid papulosis (12cases) were retrieved from the archives of Department of Pathology in Jichi Medical University, sliced, deparaffinized, and immunostained with antibodies against IL-33 (Nessy-1,Enzo Life Science, NewYork, USA), RhoA (rabbit polyclonal, proteintech, Rosemont, USA) and Ect2 (rabbit polyclonal, LifeSpan BioSciences, Seattle,USA).

The staining intensity was observed by experienced dermatologists, and classified into four categories (0; negative, 1; weak, 2; strong, 3; very strong), which was compiled, and statistically analyzed, following the literature (5).

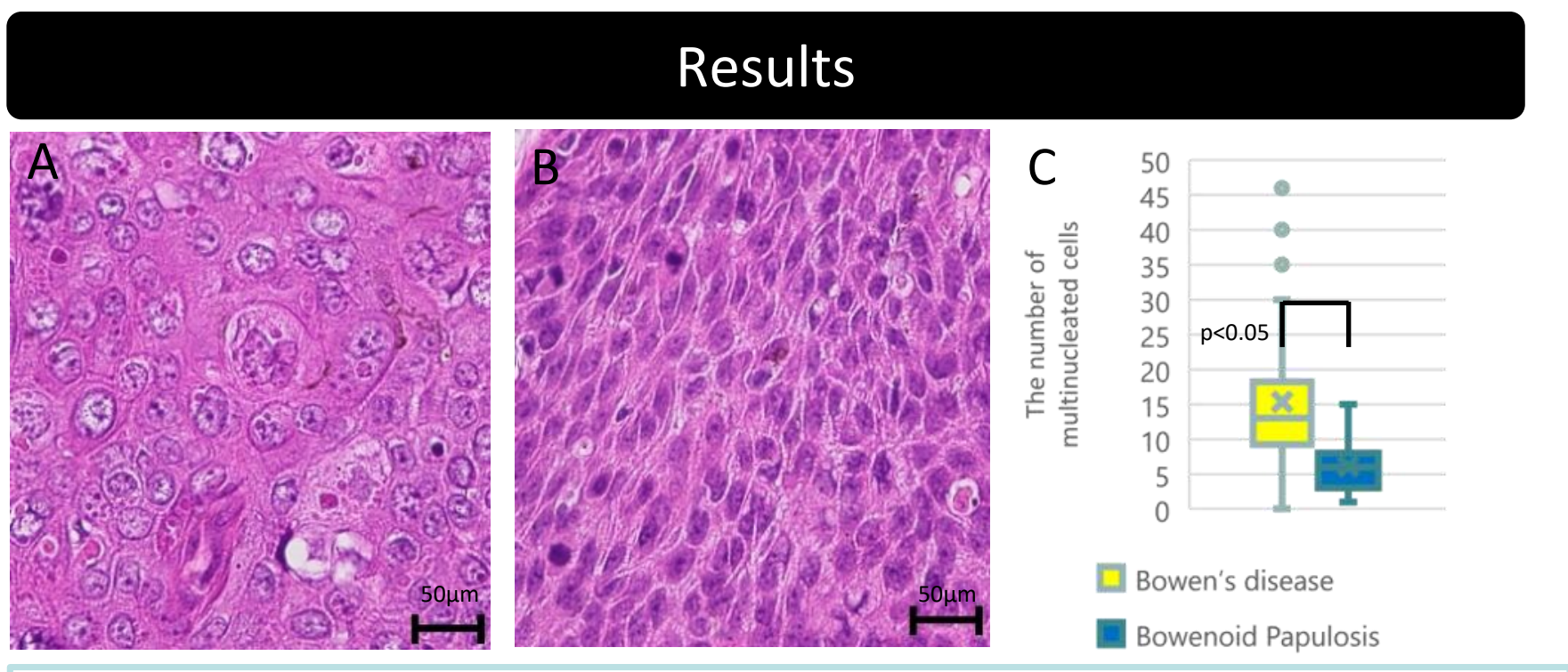

Figure 1. The number of multinucleated cells in Bowen's disease were larger (A) than that in Bowenoid papulosis (B). The number of multinucleated cells high power field was counted, and shown in the graph (C).
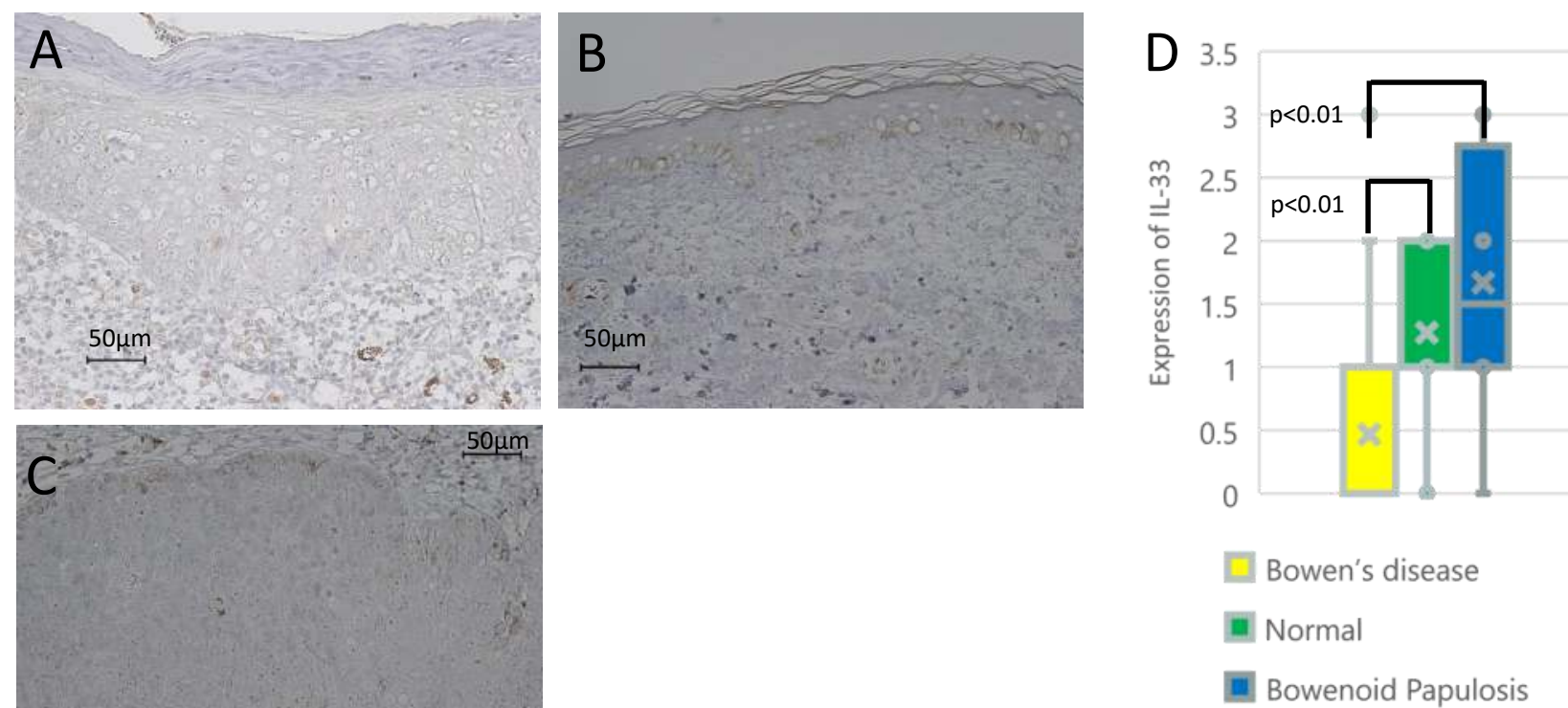

Figure 2. Expression of IL-33 was reduced in the lesional epidermis of Bowen's diseases (A) compared to surrounding normal epidermis (B), and Bowenoid papulosis (C). The staining intensity was classified into four categories $\langle 0 \sim 3\rangle$, and shown in the graph (D).
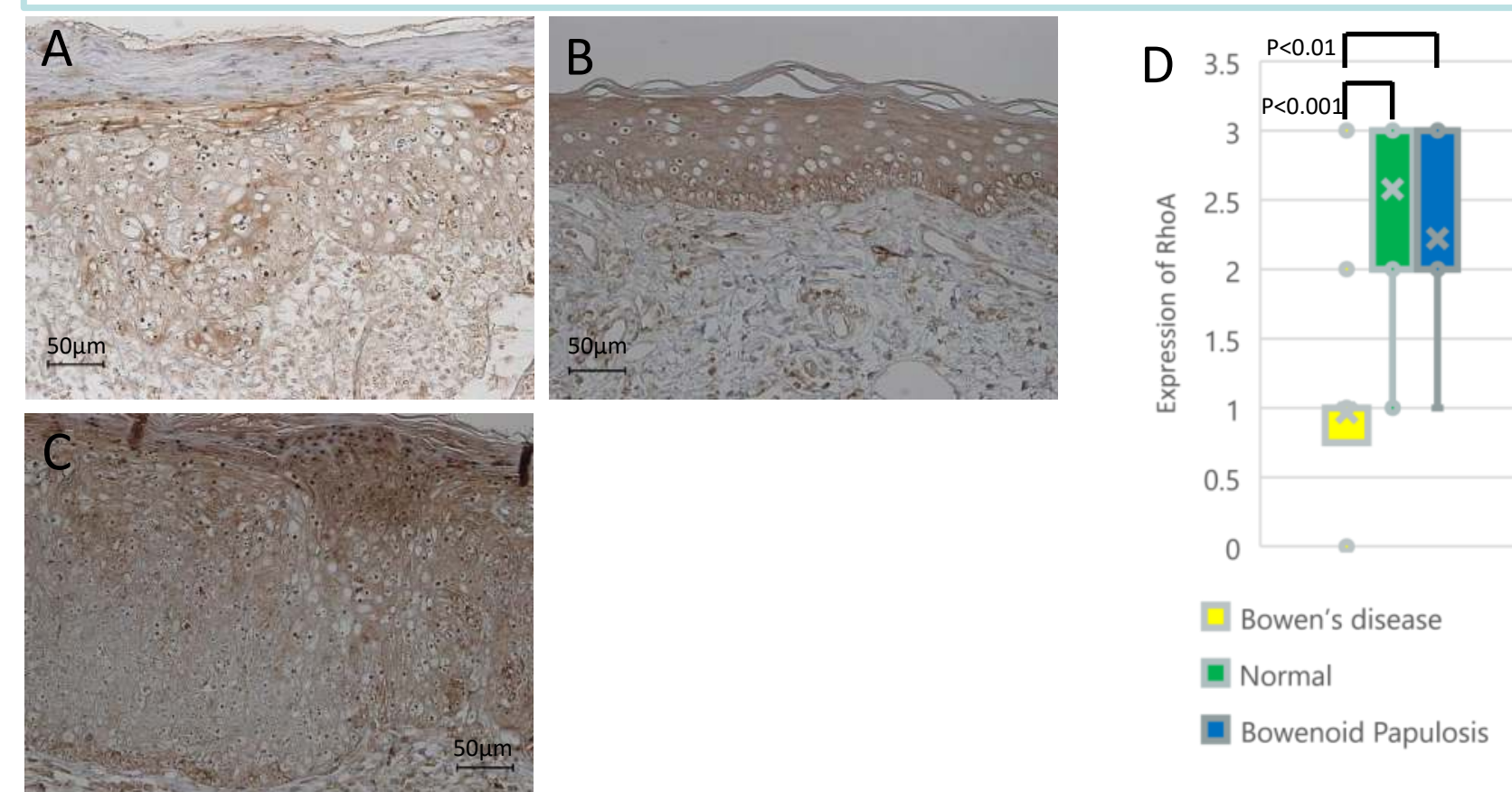

Figure 3. Expression of RhoA was reduced in the lesional epidermis of Bowen's disease $(A)$ compared to surrounding normal epidermis $(B)$, and Bowenoid papulosis $(C)$. The staining intensity was classified into four categories $\langle 0 \sim 3\rangle$, and shown in the graph (D).
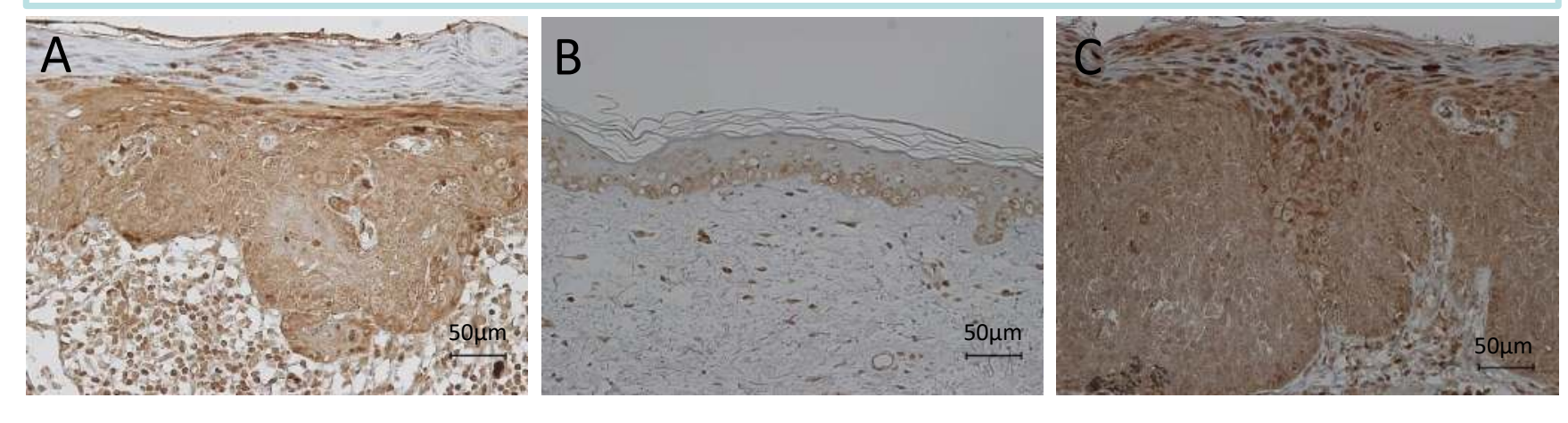

D
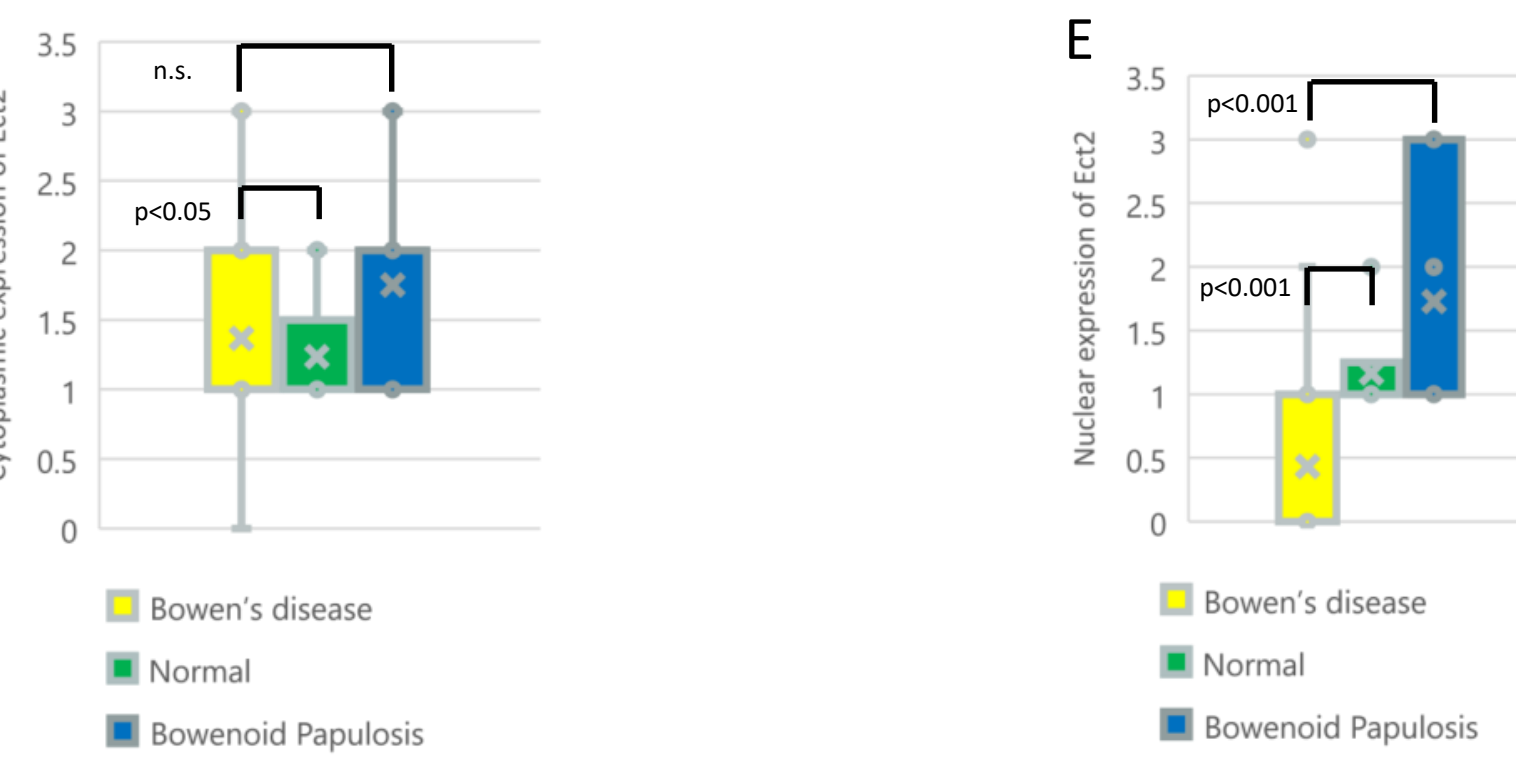

Figure 4. Cytoplasmic expression of Ect2 was comparable in the lesional epidermis of Bowen's disease $(A)$ and in the surrounding normal epidermis (B), and Bowenoid papulosis (C). Nuclear expression of Ect2 was decreased in Bowen's diseases (A) compared to surrounding normal epidermis (B) and Bowenoid papulosis (C). The staining intensity was classified into four categories $\langle 0 \sim 3\rangle$, and shown in the graph $(D, E)$.

\section{Summary and Discussion}

This study demonstrated that 1) multinucleated cells in Bowen's disease were more pronounced compared to Bowenoid papulosis, 2) expression of IL-33 and RhoA was less in Bowen's disease than that in Bowenoid papulosis. 3) nuclear expression of Ect2 was less in Bowen's disease than that in Bowenoid papulosis.

Our previous study indicated that loss of IL-33 expression in keratinocytes lead to decreased expression of RhoA and Ect2, which resulted in defect in cytokinesis. RhoA and Ect 2 play pivotal roles in cytokinesis, and the loss of expression of these molesules results in multinucleated cells in culture as previously reported.

Our study suggests that the multinucleated cells in Bowen's disease could be the result of defect in cytokinesis, which could be attributed to the decreased expression of IL-33, RhoA and Ect2 in cells. 